absent responses to vaccination against SARS-CoV2 and cannot abandon social distancing and masking precautions.

In the end, there is no advantage from prolonged maintenance in indolent lymphomas, and 2 years should remain the standard duration if maintenance rituximab is offered. The MabCute trial thus supports that extended dosing of rituximab is both too much, and also not enough to offer cure or improve overall survival. Future studies should focus on identifying the minimum number of maintenance doses needed to improve outcomes, particularly in light of a pandemic threat.

\section{Disclosures}

No conflicts of interest to disclose.

\section{References}

1. Berinstein NL, Grillo-Lopez AJ, White CA, et al. Association of serum rituximab (IDEC-C2B8) concentration and anti-tumor response in the treatment of recurrent low-grade or follicular non-Hodgkin's lymphoma. Ann Oncol. 1998;9(9):995-1001.

2. Gordan LN, Grow WB, Pusateri A, Douglas V, Mendenhall NP, Lynch JW. Phase II trial of individualized rituximab dosing for patients with CD20-positive lymphoproliferative disorders. J Clin Oncol. 2005;23(6):1096-1102.

3. Nabhan C, Ollberding NJ, Villines D, et al. A systematic review of comparative schedule-related toxicities with maintenance rituximab in follicular and mantle cell lymphomas. Leuk Lymphoma. 2014;55(6):1288-1294.
4. Salles G, Seymour JF, Offner F, et al. Rituximab maintenance for 2 years in patients with high tumour burden follicular lymphoma responding to rituximab plus chemotherapy (PRIMA): a phase 3, randomised controlled trial. Lancet. 2011;377(9759):42-51.

5. Vidal L, Gafter-Gvili A, Salles G, et al. Rituximab maintenance for the treatment of patients with follicular lymphoma: an updated systematic review and meta-analysis of randomized trials. J Natl Cancer Inst. 2011;103(23):1799-1806.

6. Rule S, Barreto W, Briones J, et al. Efficacy and safety assessment of prolonged maintenance with subcutaneous rituximab in patients with relapsed or refractory indolent non-Hodgkin lymphoma: results of the phase III MabCute study. Haematologica. 2021;107(2):500-509.

7. Taverna C, Martinelli G, Hitz F, et al. Rituximab maintenance for a maximum of 5 years after single-agent rituximab induction in follicular lymphoma: results of the randomized controlled phase III trial SAKK 35/03. J Clin Oncol. 2016;34(5):495-500.

8. Kahl BS, Hong F, Williams ME, et al. Rituximab extended schedule or re-treatment trial for low-tumor burden follicular lymphoma: Eastern Cooperative Oncology Group protocol E4402. J Clin Oncol. 2014;32(28):3096-3102.

9. Hiddemann W, Barbui AM, Canales MA, et al. Immunochemotherapy with obinutuzumab or rituximab for previously untreated follicular lymphoma in the GALLIUM study: influence of chemotherapy on efficacy and safety. J Clin Oncol. 2018;36(23):2395-2404.

10. Mato AR, Roeker LE, Lamanna N, et al. Outcomes of COVID-19 in patients with CLL: a multicenter international experience. Blood. 2020;136(10):1134-1143.

11. Herishanu Y, Avivi I, Aharon A, et al. Efficacy of the BNT162b2 mRNA COVID-19 vaccine in patients with chronic lymphocytic leukemia. Blood. 2021;137(23):3165-3173.

12. Proceedings of the COVID-19 Vaccine Panel: a Lymphoma Research Foundation White Paper. https://lymphoma.org/wpcontent/uploads/2021/04/Proceedings-of-the-COVID-19-VaccinePanel_LRF-White-Paper_April-2021.pdf; 2021. (Last accessed May 12, 2021)

\title{
An exciting RXRA mutant revives interest in retinoids for acute myeloid leukemia
}

\section{Fang Qiu' and Hugues De The ${ }^{1-3}$}

${ }^{1} I N S E R M ~ U M R$ 944, CNRS UMR 7212, Université de Paris, IRSL, Hôpital Saint Louis; ${ }^{2}$ Collège de France, Oncologie Cellulaire et Moléculaire, PSL University, INSERM UMR 1050, CNRS UMR 7241, and ' ${ }^{3}$ Department of Hematology, Hôpital Saint Louis (Assistance publique Hôpitaux de Paris) and Paris University, Paris, France

E-mail: HUGUES DE THÉ - hugues.dethe@inserm.fr

doi:10.3324/haematol.2021.279152

T he idea that "one size fits all" is obviously outdated for acute myeloid leukemia (AML) therapy: tomorrow's treatments will depend on phenotypically or genetically defined subtypes. The most striking example is acute promyelocytic leukemia (APL), driven by the PMLRARA fusion protein. In APL, a subtype that accounts for $5 \%$ of cases of AML, a combination of two targeted agents, all trans-retinoic acid (ATRA) and arsenic trioxide, cures over $90 \%$ of patients through PML-RARA driver degradation, differentiation and restoration of PML-dependent senescence. ${ }^{1}$ These clinical successes have spurred attempts to harness the power of retinoids in other cancers. Unfortunately, ATRA treatment alone remains poorly effective in most non-APL AML. ${ }^{2}$

Retinoid signaling is complex and still incompletely understood. ${ }^{3}$ ATRA acts primarily through heterodimeric complexes of retinoic acid receptors (RAR) assembled with retinoid X receptors (RXR). These RXR are key heterodimerization partners of many class-II nuclear receptors and may be ligand-dependent transcription factors or silent receptors, allowing sequence-specific DNA recognition. ${ }^{4}$ Hence, therapeutic targeting of RXR could be a strategy to activate targets under the control of the RXR/RAR transcriptional complex. However, in principle, RXR/RAR signaling cannot be activated by RXR ligands alone, at least in part because co-repressors remain firmly bound to RAR. This may be modulated by other signaling cascades/second messengers, such as cAMP. ${ }^{5}$

In AML, this simple view has been challenged. RXR ligands (rexinoids, such as bexarotene) may exert some differentiating effects ex vivo and in vivo, 5,6 Hematopoietic cells and some AML express endogenous RXRA ligands. ${ }^{7,8}$ Two recent studies have revived interest in RXRA signaling in AML. The first demonstrated that, in AML driven by KMT2A-MLLT3, rexinoids partially suppressed AML growth and triggered differentiation. ${ }^{8}$ Moreover, genetic ablation of RXR accelerated AML growth, while concomitant activation of both RXRA and RARA precipitated differentiation or apoptosis. It is hoped that dual activation of these key regulators may harness retinoids more effi- 

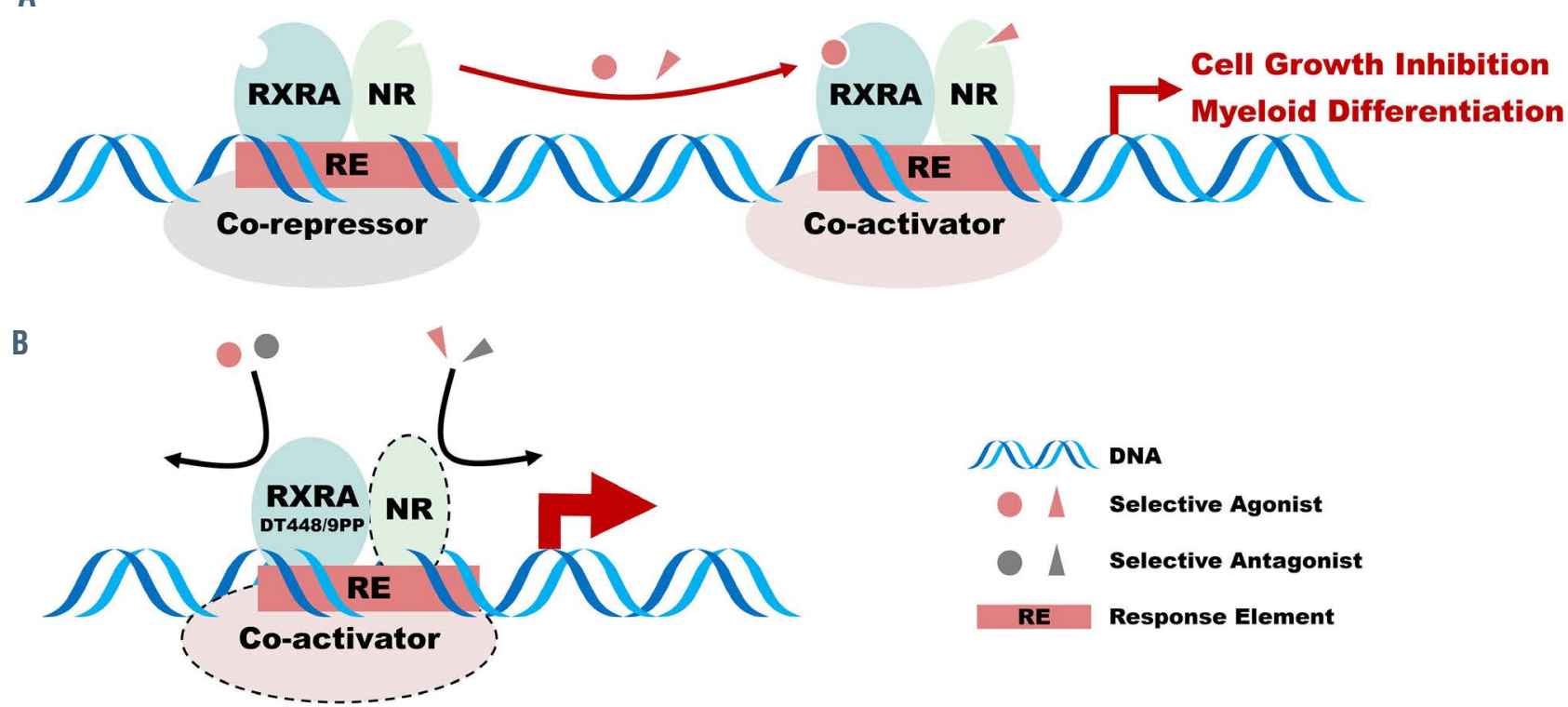

Figure 1. Schematic summary of the effects of constitutively active RXRA DT448/9PP. (A) Under normal circumstances, the transcriptional activity of RXRA heterodimerized with other nuclear receptors (NR), including RARA, remains silent, because of co-repressor binding. Selective agonists activate RXRA/NR-driven transcription, resulting in cellular differentiation and growth arrest. (B) Mutations of residues 488/9 in RXRA allow potent ligand-independent transcriptional activation and drive differentiation.

ciently in AML. ${ }^{2}$ In the second study, published last year in Haematologica, di Martino et al. report a serendipitously identified activating mutation in RXRA (RXRA DT448/9PP), which potently activates rexinoid/retinoid downstream signaling and suffices to induce terminal differentiation of KMT2A-MLLT3-transformed cells. ${ }^{9}$ The Cterminal helix 12 or AF-2 helix of RXRA, is a critical determinant of ligand-dependent transcriptional activity through control of co-activator/co-repressor binding. Surprisingly, di Martino et al. demonstrated that RXRA DT448/9PP overexpression resulted in enhanced transcriptional activity leading to multiple features of differentiation, notably loss of colony-forming ability, in KMT2AMLLT3-transformed AML cells. Amazingly, this constitutively active RXRA variant binds co-activators completely independently of ligands. Accordingly, transactivation could not be abrogated or further boosted by selective antagonists of RXR or other nuclear receptors, or their agonists, respectively (Figure 1).

These intriguing observations imply that even though rexinoids and retinoids synergize for myeloid differentiation of those AML, ${ }^{8}$ more profound "unconventional" activation by RXRA can initiate terminal differentiation. This master transcriptional regulatory complex deserves further studies to mechanistically decipher how it can become so potent in the absence of ligands. Issues of partner proteins, post-translational modifications or non-coding RNA, all come to mind. Whatever the molecular mechanism, these observations suggest that the RXRA/RARA axis, when super-activated, has the potential to initiate terminal differentiation of some AML cells. Further studies should determine which AML exhibit this exquisite sensitivity to RXRA signaling. This re-emerging theme of retinoid sensitivity in non-APL $\mathrm{AML}^{2}$ could be particularly important in the context of combinations of treatment, particularly with decitabine, as encouraging clinical trials have been published recently, ${ }^{10}$ with more likely to come.

\section{Disclosures \\ No conflicts of interest to disclose.}

\section{References}

1. de Thé H, Pandolfi PP, Chen Z. Acute promyelocytic leukemia: a paradigm for oncoprotein-targeted cure. Cancer Cell. 2017;32(5):552-560.

2. Geoffroy MC, Esnault C, de Thé H. Retinoids in hematology: a timely revival? Blood. 2021;137(18):2429-2437.

3. de Thé $H$. Differentiation therapy revisited. Nat Rev Cancer. 2018;18(2):117-127.

4. De Bosscher K, Desmet SJ, Clarisse D, Estébanez-Perpiña E, Brunsveld L. Nuclear receptor crosstalk-defining the mechanisms for therapeutic innovation. Nat Rev Endocrinol. 2020;16(7):363-377.

5. Altucci L, Rossin A, Hirsch O. Rexinoid-triggered differentiation and tumor-selective apoptosis of acute myeloid leukemia by protein kinase A-mediated desubordination of retinoid X receptor. Cancer Res. 2005;65(19):8754-8765.

6. Sanchez PV, Glantz ST, Scotland S, Kasner MT, Carroll M. Induced differentiation of acute myeloid leukemia cells by activation of retinoid X and liver X receptors. Leukemia. 2014;28(4):749-760.

7. Niu H, Fujiwara H, di Martino O. Endogenous retinoid X receptor ligands in mouse hematopoietic cells. Sci Signal. 2017;10(503):eaan1011.

8. Di Martino O, Niu H, Hadwiger G. Endogenous and combination retinoids are active in myelomonocytic leukemias. Haematologica. 2021;106(4):1008-1021

9. Di Martino O, Ferris MA, Hadwiger G, et al. RXRA DT448/9PP generates a dominant active variant capable of inducing maturation in acute myeloid leukemia cells. Haematologica. 2021;107(2):417-426.

10. Lübbert M, Grishina O, Schmoor C. Valproate and retinoic acid in combination with decitabine in elderly nonfit patients with acute myeloid leukemia: results of a multicenter, randomized, $2 \times 2$, phase II trial. J Clin Oncol. 2020;38(3):257-270. 